\title{
FATORES DE RISCO RELACIONADOS À PNEUMONIA NO PÓS-OPERATÓRIO PRECOCE DE TRANSPLANTE DE FÍGADO
}

\author{
Risk factors associated pneumonia in post operative early liver transplant \\ Lorena Guedes Bravo1, Gustavo Rêgo Coelho¹, Maria Flávia Amâncio Campos¹, Evelyne Santana Girão', \\ José Huygens Parente Garcia ${ }^{1}$
}

\section{RESUMO}

Objetivo: Identificar os principais fatores de risco relacionados à pneumonia no pós-operatório precoce de transplante de fígado. Métodos: Foi realizado um estudo de caso controle no período de junho a dezembro de 2014, com pacientes submetidos a transplante hepático nesse período, em um hospital universitário de Fortaleza/CE. Foram incluídos no estudo, pacientes de ambos os gêneros que foram submetidos a transplante hepático, e excluídos aqueles que apresentavam dados insuficientes no prontuário, prejudicando a análise dos dados, os que evoluíram para óbito até $05^{\circ}$ dia pós-operatório e os que foram submetidos a retransplante por disfunção primária do enxerto. A coleta de dados ocorreu de junho a dezembro de 2014, através da análise prospectiva dos prontuários de todos os pacientes submetidos a transplante hepático nesse período. Os prontuários foram analisados no período pré, trans e pós-operatório até a alta hospitalar. Resultados: A amostra foi composta por 52 pacientes, sendo $69,2 \%$ do gênero masculino, com idade média de $55( \pm 12,5)$ anos. Foram encontradas diferentes etiologias, das quais as de maior prevalência foram as hepatites virais $(51,9 \%)$ e a cirrose alcoólica $(21,1 \%)$. Os participantes da amostra apresentaram escore MELD médio $22( \pm 4,8)$. Os potenciais fatores de risco para o desenvolvimento de pneumonia precoce no pós-transplante de fígado através da análise bivariada incluem idade $(p=0,09)$, MELD pré-operatório $(p=0,22)$, internamento prévio à cirurgia $(p=0,09)$, tempo de isquemia fria $(p=0,22)$ e tempo de ventilação mecânica $(p=0,08)$. Na análise multivariada, os fatores de risco independentes foram: maior tempo em unidade de terapia intensiva $(p=0,002)$, necessidade do procedimento de reintubação $(p=0,002)$ e uso de antibiótico profilático diferente de Ampicilina + Sulbactam (antibiótico profilático de rotina do serviço) $(p=0,02)$. Conclusão: A pneumonia é uma complicação pulmonar frequente, com alta taxa de mortalidade no pós-operatório precoce de transplante hepático. Essa complicação pode ser associada a vários fatores pré, trans e pós-operatório.

Descritores: Unidade de Terapia Intensiva; Transplante de Fígado; Diafragma; Respiração Artificial; Extubação.

Instituições:

Departamento de Cirurgia do Hospital Universitário Walter Cantídio - Fortaleza/CE - Brasil

\section{Correspondência:}

Lorena Guedes Bravo

Centro de Transplante de Fígado do Ceará

Rua Capitão Francisco Pedro, 1290 - CEP 60430-370 - Fortaleza/ CE - Brasil

Tel: (85) 9626-0800

Email: Iorenaguedesb@hotmail.com

Recebido em: 15/01/2015
Aceito em: 19/02/2015

\section{INTRODUÇÃO}

O transplante é considerado uma das cirurgias mais complexas da medicina moderna, devido às suas peculiaridades com relação à técnica em si e aos cuidados pré e pós-operatórios que exigem adesão a um plano terapêutico em longo prazo, o acompanhamento multiprofissional contínuo e o uso de medicações imunossupressoras. ${ }^{1}$

De acordo com o Registro Brasileiro de Transplantes (RBT), no ano de 2013, o Brasil foi o segundo em número absoluto de transplante de fígado entre 30 países, com o total de 1.723 procedimentos realizados, perdendo apenas para os Estados Unidos. Segundo a mesma fonte, no ano de 2014 foram realizados no Brasil um total de 1.755 transplantes de fígado, com 1.605 de doador 
falecido e 150 de doador vivo. O estado do Ceará ocupa o primeiro lugar no ranking nacional de Transplantes de fígado por milhão de população por estado. ${ }^{2}$

Entre cinco e dez anos, a taxa de sobrevida póstransplante é cerca de $70 \%$, o que leva a uma maior preocupação com a qualidade de vida desses pacientes, após a cirurgia. ${ }^{3}$

Atualmente, o transplante de fígado representa o tratamento mais eficiente para pacientes portadores de doença hepática terminal, pois trata-se de um método capaz de reverter o quadro terminal onde não há outra possibilidade de tratamento. ${ }^{4-6}$

A significativa melhora na sobrevida resultou em aumento progressivo do número de transplantes de fígado realizados em vários países. Porém, o tempo em lista de espera de pacientes aguardando esse procedimento aumentou mais rapidamente, principalmente devido à maior procura dos pacientes nos serviços de transplante. ${ }^{7}$

Apesar do aperfeiçoamento das técnicas operatórias, profilaxia antimicrobiana, imunossupressão, anestesia e cuidados intensivos, ainda é comum a presença de complicações pulmonares, como a pneumonia (PNM), atelectasia e o derrame pleural, durante a permanência do paciente na unidade de terapia intensiva (UTI). ${ }^{8}$

Este estude teve como objetivo identificar os principais fatores de risco relacionados à pneumonia no pósoperatório precoce de transplante de fígado.

\section{MÉTODOS}

Trata-se de um estudo de caso controle, realizado em um Hospital Universitário de Fortaleza - Ceará. Foram incluídos no estudo pacientes de ambos os gêneros que foram submetidos a transplante hepático e excluídos aqueles que apresentavam dados insuficientes no prontuário que prejudicassem a análise dos dados, os que evoluíram para o óbito até $05^{\circ}$ pós-operatório e os que foram submetidos à retransplante por disfunção primária do enxerto.

O projeto de pesquisa foi aprovado pelo comitê de ética em pesquisa do Hospital Universitário Walter Cantídio com o parecer de número 839.839 e seguiu os preceitos éticos de pesquisa em seres humanos regulamentadas pela resolução $n^{\circ} 466 / 12$ do Conselho Nacional de Saúde/ Ministério da Saúde. ${ }^{9}$

A coleta de dados ocorreu de junho a dezembro de 2014, através da análise prospectiva dos prontuários de todos os pacientes submetidos a transplante hepático nesse período. Os dados foram transcritos para uma ficha de coleta de dados elaborada pelos pesquisadores, com os dados constantes nos registros da equipe multiprofissional referentes às variáveis (idade, gênero, Score MELD, internamento prévio, uso de antibiótico profilático, tabagismo, comorbidades, reintubação, fisioterapia pré-operatória, transfusão de hemocomponentes, tempo de cirurgia, tempo de isquemia fria, tempo de isquemia quente, tempo de ventilação mecânica, tempo em UTI) envolvidas na pesquisa. Os prontuários foram analisados no período pré, trans e pós-operatório até a alta hospitalar.

Após a análise dos prontuários, os pacientes foram divididos em dois grupos: os que apresentaram pneumonia (Grupo Pneumonia) e os que não apresentaram pneumonia (Grupo Controle). A pneumonia foi caracterizada como: novo e persistente infiltrado no raio $X$ sugestivo de pneumonia associado a, pelo menos, dois fatores: aumento e mudança do aspecto de secreção traqueal, aumento do número de leucócitos $>12.000 \mathrm{cel} / \mathrm{ml}$ ou $<4.000 \mathrm{cel} / \mathrm{ml}$, hipertermia - febre $\left(\mathrm{T}>38^{\circ} \mathrm{C}\right.$ ), hipotermia $\left(\mathrm{T}<35^{\circ} \mathrm{C}\right)$ e piora ventilatória/ dispneia calculada através do índice de Oxigenação (PaO2/FiO2)..$^{10}$

As informações foram agrupadas e formatadas em um banco de dados para análise no programa Stata versão 12 (Stata Corporation, College Station). As variáveis categóricas foram analisadas como frequência absoluta e relativa e as variáveis contínuas como média e desviopadrão. Para analisar as associações entre as variáveis categóricas foi utilizado o teste de qui-quadrado e para as variáveis contínuas o teste t-Student (análise bivariada). Para avaliar os fatores associados à PNM, foi utilizado um modelo de regressão logística multivariado em que a ocorrência de pneumonia foi considerada como variável dependente. Os fatores pré, trans e pósoperatório de transplante de fígado foram considerados como variáveis independentes.

A análise multivariada foi realizada considerando $p<0,20$ para incluir a variável no modelo logístico, e $p<0,05$ para manter a variável no modelo final. As variáveis que apresentaram colinearidade não foram incluídas no modelo final. A odds ratio (OR) bruta e ajustada foi usada para analisar os fatores de associação com o desfecho, e foi considerada significativamente diferente quando o intervalo de confiança de $95 \%$ não incluiu o valor 1. Fatores de confusão e interação também foram investigados, e quando identificados, foram mantidos no modelo mesmo não apresentando significância de $p<0,05$. $^{11}$

\section{RESULTADOS}

Foram avaliados sete indivíduos, tendo sido excluídos A amostra foi composta por 52 pacientes, sendo $69,2 \%$ $(n=36)$ do gênero masculino e $30,8 \%(n=16)$ do gênero feminino, com idade média de $55( \pm 12,5)$ anos. 
Foram encontradas diferentes etiologias (Tabela 1), dasquais as de maior prevalência foram as hepatites virais $(51,9 \%)$ e a cirrose alcoólica $(21,1 \%)$.

Tabela 1 -Etiologia dos pacientes submetidos a transplante hepático de Fortaleza - Ceará. $n=52$

\begin{tabular}{llc}
\hline \multicolumn{1}{c}{ Etiologia } & N & Percentual (\%) \\
\hline Cirrose por Vírus C + Hepatocarcinoma & 11 & 21,2 \\
Cirrose por Vírus C & 9 & 17,3 \\
Cirrose por Vírus B + Hepatocarcinoma & 2 & 3,8 \\
\hline Hepatite auto imune & 5 & 9,6 \\
\hline Cirrose Alcoólica & 8 & 15,4 \\
Cirrose criptogênica & 3 & 5,8 \\
Cirrose alcóolica + Sd hepatopulmonar & 1 & 1,9 \\
Cirrose Biliar Primária & 1 & 1,9 \\
Tumor Neuroendocrino & 1 & 1,9 \\
Cirrose por Vírus B & 1 & 1,9 \\
Colangite de repetição & 1 & 1,9 \\
Polineuropatia Amilodótica Familiar & 1 & 1,9 \\
Cirosse Alccolica + hemocromatose & 2 & 3,8 \\
Cirrose por virus C + Sd hepatorrenal & 4 & 7,7 \\
\hline Doença de Wilson & 1 & 1,9 \\
Cirrose Biliar Secundaria (síndrome & 1 & 1,9 \\
colestática) & & \\
\hline
\end{tabular}

Em relação à procedência (Tabela 2), a maior prevalência foi a da região Nordeste $(65,3 \%)$, seguida da região Norte, Sudeste e Sul.

Tabela 2 -Valores do RMS com e sem a utilização de pressão positiva expressos em média e desvio padrão

\begin{tabular}{lcc}
\hline \multicolumn{1}{c}{ Origem } & N & Percentual \\
\hline Ceará & 18 & 34,6 \\
Piauí & 6 & 11,5 \\
Amazonas & 4 & 7,7 \\
Maranhão & 2 & 3,8 \\
São Paulo & 5 & 9,6 \\
Pará & 5 & 9,6 \\
Acre & 2 & 3,8 \\
Roraima & 1 & 1,9 \\
\hline Paraná & 1 & 1,9 \\
\hline
\end{tabular}

Os participantes da amostra apresentaram escore MELD corrigido médio de $22( \pm 4,8) .48 \%(n=25)$ pertenciam ao grupo sanguíneo $A, 36,5 \%(n=19)$ ao grupo $0,13,5 \%$ $(n=7)$ ao $B$ e $1,9 \%(n=1)$ ao $A B$.

Após o transplante, $34,6 \%(n=18)$ dos pacientes da amostra apresentaram pneumonia.

A tabela 3 apresenta a análise bivariada entre os fatores pré, trans e pós-operatório de transplante de fígado e o desenvolvimento de PNM. As variáveis elegíveis para o modelo final foram: idade, antibiótico profilático, reintubação, transfusão de hemocomponentes, tempo de isquemia fria, tempo de ventilação mecânica, tempo na UTI, MELD e internamento prévio.

Tabela 3 - Análise bivariada entre pneumonia e fatores pré, trans e pós-operatório de transplante de fígado

\begin{tabular}{|c|c|c|c|c|c|}
\hline \multirow[b]{2}{*}{ Variáveis } & \multicolumn{2}{|c|}{ Grupo } & \multirow[b]{2}{*}{ Total } & \multirow[b]{2}{*}{ OR } & \multirow[b]{2}{*}{$P$} \\
\hline & $\begin{array}{l}\text { Pneumonia } \\
(n=18)\end{array}$ & $\begin{array}{c}\text { Controle } \\
(\mathrm{n}=34)\end{array}$ & & & \\
\hline Idade & $\begin{array}{c}59,5 \pm 7,82 \\
\text { anos }\end{array}$ & $\begin{array}{l}52,97 \pm 14,04 \\
\text { anos }\end{array}$ & - & $\begin{array}{c}1,05 \\
(0,99-1,12)\end{array}$ & $0,09 \dagger$ \\
\hline $\begin{array}{l}\text { Sexo } \\
\text { Feminino } \\
\text { Masculino }\end{array}$ & $\begin{array}{c}6(37.5 \%) \\
12(33,3 \%)\end{array}$ & $\begin{array}{l}10(62,5 \%) \\
24(66,7 \%)\end{array}$ & $\begin{array}{l}16(100,0 \%) \\
36(100,0 \%)\end{array}$ & $\begin{array}{c}1 \\
0,83(0,24-2,84)\end{array}$ & $\begin{array}{c}- \\
0,77\end{array}$ \\
\hline \multicolumn{6}{|l|}{$\begin{array}{l}\text { Antibiótico } \\
\text { Profilático }\end{array}$} \\
\hline $\begin{array}{l}\text { Ampicilina } \\
+ \text { Sulbactam }\end{array}$ & $10(25,6 \%)$ & $29(74,4 \%)$ & $39(100,0 \%)$ & 1 & - \\
\hline Outros & $8(61,5 \%)$ & $5(38,55)$ & $13(100,0 \%)$ & $4,64(1,22-17,51)$ & $0,02^{*}$ \\
\hline \multicolumn{6}{|l|}{ Tabagismo } \\
\hline Não & $9(36,0 \%)$ & $1(64,0 \%)$ & $25(100,0 \%)$ & 1 & - \\
\hline Sim & $9(33,3 \%)$ & $18(66,7 \%)$ & $27(100,0 \%)$ & $0,89(0,28-2,78)$ & 0,84 \\
\hline \multicolumn{6}{|l|}{ Comorbidades } \\
\hline Não & $8(32,0)$ & $17(68,0 \%)$ & $25(100,0 \%)$ & 1 & - \\
\hline Sim & $10(37,0 \%)$ & $17(63,0 \%)$ & $27(100,0 \%)$ & $1,25(0,39-3,93)$ & 0,70 \\
\hline \multicolumn{6}{|l|}{ Reintubação } \\
\hline Não & $9(21,4 \%)$ & $33(78,6 \%)$ & $42(100,0 \%)$ & 1 & - \\
\hline Sim & $9(90,0 \%)$ & $1(10,0 \%)$ & $10(100 \%)$ & $33(3,68-295,9)$ & $0,002^{*}$ \\
\hline \multicolumn{6}{|l|}{$\begin{array}{l}\text { Transfusão de } \\
\text { hemocomponentes }\end{array}$} \\
\hline Não & $3(16,7 \%)$ & $15(83,3 \%)$ & $18(100,0 \%)$ & $3.94(0,96-16,20)$ & $0,05^{*}$ \\
\hline Sim & $15(44,1 \%)$ & $19(55,9 \%)$ & $34(100,0 \%)$ & 1 & - \\
\hline $\begin{array}{l}\text { Fisioterapia no } \\
\text { Pré-Operatório }\end{array}$ & $\begin{array}{c}1,50 \pm 1,88 \\
\text { atendimentos }\end{array}$ & $\begin{array}{c}1,24 \pm 1,43 \\
\text { atendimentos }\end{array}$ & - & $1,11(0,77-1,58)$ & 0,56 \\
\hline $\begin{array}{l}\text { Tempo de } \\
\text { Cirurgia }\end{array}$ & $\begin{array}{c}6,06 \pm 1,21 \\
\text { horas }\end{array}$ & $\begin{array}{c}6,24 \pm 1.35 \\
\text { horas }\end{array}$ & - & $0,89(0,56-1,41)$ & 0,63 \\
\hline $\begin{array}{l}\text { Tempo de } \\
\text { Isquemia Fria }\end{array}$ & $\begin{array}{l}5,72 \pm 1,90 \\
\text { horas }\end{array}$ & $\begin{array}{c}5,12 \pm 1,53 \\
\text { horas }\end{array}$ & - & $1,24(0,88-1,75)$ & $0,22 \dagger$ \\
\hline $\begin{array}{l}\text { Tempo de } \\
\text { Isquemia Quente }\end{array}$ & $\begin{array}{c}32,67 \pm 5,38 \\
\text { minutos }\end{array}$ & $\begin{array}{c}33,09 \pm 6,49 \\
\text { minutos }\end{array}$ & - & $0,98(0,89-1,08)$ & 0,81 \\
\hline $\begin{array}{l}\text { Tempo de } \\
\text { Ventilação } \\
\text { Mecânica }\end{array}$ & $\begin{array}{c}55,17 \pm 82,32 \\
\text { horas }\end{array}$ & $\begin{array}{l}20,97 \pm 43,86 \\
\text { horas }\end{array}$ & - & $1,01(0,99-1,02)$ & $0,08 \dagger$ \\
\hline $\begin{array}{l}\text { Tempo na } \\
\text { Unidade de } \\
\text { Terapia Intensiva }\end{array}$ & $\begin{array}{c}8,89 \pm 4,77 \\
\quad \text { dias }\end{array}$ & $\begin{array}{l}3,15 \pm 1,58 \\
\quad \text { dias }\end{array}$ & - & $2,26(1,34-3,82)$ & $0,002^{*}$ \\
\hline MELD & $23,28 \pm 3,08$ & $21,56 \pm 5,50$ & - & $1,08(0,95-1,22)$ & $0,22 \dagger$ \\
\hline $\begin{array}{l}\text { Internamente } \\
\text { Prévio }\end{array}$ & $7,83 \pm 14,67$ & $1,97 \pm 5,25$ & - & $1,08(0,98-1,18)$ & $0,09 \dagger$ \\
\hline
\end{tabular}

A tabela 4 apresenta o modelo final de regressão logística multivariada dos fatores associados à pneumonia após transplante de fígado. Com relação ao antibiótico profilático, qualquer outra substância ou combinação de drogas diferente da Ampicilina + Sulbactam, apresenta 7,68 vezes mais chance de desenvolver pneumonia. Pacientes que precisam 
realizar o procedimento de reintubação possuem 26,06 vezes mais chance de adquirir pneumonia. Os pacientes com pneumonia permaneciam em média $8,89 \pm 4,77$ dias na UTI, enquanto que os pacientes controles ficaram apenas $3,15 \pm 1,58$ dias $(p<0,001)$.

Tabela 4 - Regressão logística multivariada dos fatores associados a pneumonia após transplante de fígado

\begin{tabular}{|c|c|c|c|}
\hline \multicolumn{2}{|c|}{ Variáveis } & \multirow{2}{*}{$\frac{\text { OR Bruta }}{1}$} & \multirow{2}{*}{$\begin{array}{c}\text { OR Ajustada } \\
1\end{array}$} \\
\hline $\begin{array}{l}\text { Antibiótico } \\
\text { Profilático }\end{array}$ & $\begin{array}{l}\text { Ampicilina }+ \\
\text { Sulbactam }\end{array}$ & & \\
\hline$(\mathrm{p}=0,002)$ & Outros & $4,64(1,22-17,51)$ & $7,68(1,01-58,33)$ \\
\hline Reintubação & Não & 1 & 1 \\
\hline$(p=0,002)$ & Sim & $33(3,68-295,9)$ & $26,06(1,30-522,13)$ \\
\hline $\begin{array}{l}\text { Dias na UTI } \\
(p=0,02)\end{array}$ & & $2,26(1,34-3,82)$ & $2,02(1,14-3,58)$ \\
\hline
\end{tabular}

Os pacientes que desenvolveram pneumonia no pós-operatório imediato de transplante hepático apresentaram uma sobrevida hospitalar de $72,2 \%$, enquanto os pacientes do grupo controle a sobrevida hospitalar foi de $97,7 \%$.

\section{DISCUSSÃO}

O transplante de fígado é uma cirurgia abdominal superior, que envolve um extenso campo de operação e um longo tempo de cirurgia. A incisão cirúrgica secciona os músculos reto e oblíquos do abdômen, sendo estes associados à respiração. ${ }^{12}$

Os indivíduos submetidos à cirurgia no abdome superior são mais propensos à disfunção diafragmática, o que resulta em uma redução na capacidade vital e na capacidade residual funcional. Além disso, a utilização de anestésicos e o efeito inibitório da dor da ferida operatória sobre a tosse e a remoção de muco, normalmente contribui para o desenvolvimento de complicações pulmonares pós-operatórias. ${ }^{13}$

Os pacientes submetidos a transplante hepático têm um maior risco para o desenvolvimento de infecção hospitalar, devido ao procedimento cirúrgico complexo, necessidade de múltiplos dispositivos invasivos, de cuidados intensivos, imunossupressão entre outros fatores. ${ }^{14}$

A prevalência média de $34 \%$ dos pacientes que apresentaram PNM no pós-transplante hepático desta pesquisa foi semelhante à de outros estudos..$^{15-18}$

Neste estudo, $44,1 \%$ dos pacientes que apresentaram pneumonia receberam transfusão de hemocomponentes até $07^{\circ}$ dia pós-transplante, sendo considerado um dos potenciais fatores de risco para o desenvolvimento de infecção pulmonar $(p=0,05)$.
Benson et al ${ }^{19} \mathrm{em}$ um estudo com o objetivo de descrever a incidência de infecção e lesão pulmonar aguda no pós-operatório de transplante de fígado, relacionada à transfusão e a associação entre hemoderivados, relataram que a pneumonia nosocomial foi um dos tipos de infecção mais comum no pós-transplante hepático nos indivíduos que foram hemotransfundidos.

Em outro estudo realizado por Pirat et al ${ }^{20}$, a PNM foi uma das complicações pulmonares mais frequentes no pós-operatório imediato de fígado ( $<30$ dias). Esses autores relataram que os pacientes que desenvolveram complicações pulmonares eram significativamente mais velhos e os que mais realizaram transfusões no intra-operatório. Nos pacientes que desenvolveram pneumonia, houve mortalidade significativamente maior, sendo associada a um mau prognóstico.

Um estudo retrospectivo realizado por Huang et al ${ }^{13}$ mostrou que os pacientes internados que necessitavam de suporte ventilatório no pré-transplante, que apresentavam comorbidades, e os que receberam 0 órgão de um doador falecido apresentavam fatores de risco para o desenvolvimento de complicações pulmonares pós-operatórias.

Glanemann et al ${ }^{12}$ realizaram um estudo através da análise de prontuários de 546 pacientes que foram submetidos a transplante hepático, com o objetivo de analisar a incidência e as indicações de reintubação nesse período. Esses autores relataram que a reintubação foi realizada principalmente devido a complicações pulmonares, entre elas a pneumonia, e que esse procedimento teve influência considerável sobre a recuperação pós-operatória do paciente, tendo sido associado a uma taxa significativamente maior de mortalidade em relação àqueles que não foram reintubados.

Sangramento intraoperatório volumoso, encefalopatia grave e exsudato pleural difuso são frequentemente associados ao retardo do desmame da ventilação mecânica, contribuindo para o desenvolvimento da infecção. ${ }^{21}$ Fatores relacionadas à prótese endotraqueal, à quantidade de transfusão de sangue intraoperatório, tempo de isquemia mais longo e não função do enxerto são outros fatores de risco importantes que predispõem a infecções do trato respiratório. ${ }^{22}$

Neste estudo, os pacientes que desenvolveram pneumonia apresentaram um escore MELD corrigido de 23, diferente do encontrado por Lin et al ${ }^{23} \mathrm{em}$ um estudo que teve como objetivo identificar os fatores preditores para complicações pulmonares. O escore MELD préoperatório $\geq 25$, a administração de fluidos e transfusão de sangue no intra-operatório podem ser preditores de complicações pulmonares, dentre elas, a pneumonia, no pós-operatório de transplante de fígado. 
Lin et al ${ }^{23}$ também demonstraram que maior tempo em unidade de terapia intensiva (UTI) foi um potencial fator de risco para o desenvolvimento de infecção, assim como demostrado também por Golfieri et al. ${ }^{18}$

Neste estudo, o tempo em UTI foi considerado como fator independente para o desenvolvimento de pneumonia $(p=0,002)$.

A prevenção de infecções é questão importante nos pacientes submetidos a transplante hepático. Elas podem estar relacionadas ao doador e/ou receptor, podem ser adquiridas na comunidade ou no ambiente hospitalar. ${ }^{24}$

O estudo de Classen et al ${ }^{25}$ mostrou que houve menores taxas de infecção quando o antibiótico foi dado até duas horas antes da incisão cirúrgica, em 1218 cirurgias acompanhadas.

No presente estudo, os pacientes que não utilizaram o esquema de profilaxia com Ampicilina + Sulbactam, devido à necessidade de antibioticoterapia no internamento prévio à cirurgia, apresentaram significativo fator de risco $(p=0,02)$ para o desenvolvimento de pneumonia após o transplante $(\mathrm{OR}=7,68)$.

As complicações pulmonares possuem um significativo efeito na morbimortalidade no pós-operatório de transplante hepático. ${ }^{26}$ Bozbas et al ${ }^{27}$ avaliaram os fatores de risco para complicações pulmonares e a taxa de mortalidade relacionada a essas complicações. Apesar dos avanços da Medicina, as complicações infecciosas ainda são frequentes e uma das principais causas de morte. A PNM grave em pacientes submetidos a esse tipo de procedimento é uma condição perigosa com significativa mortalidade.

No estudo realizado por Lin et al ${ }^{23}$, a mortalidade foi significativamente maior nos pacientes que desenvolveram pneumonia do que em indivíduos que apresentaram outras complicações pulmonares.

\section{CONCLUSÃO}

A pneumonia é uma complicação pulmonar frequente e com alta taxa de mortalidade no pós-operatório precoce de transplante hepático. Essa complicação pode ser associada a vários fatores pré, trans e pósoperatório. Os fatores de risco independentes para o desenvolvimento de PNM podem ser: tempo em UTI, necessidade do procedimento de reintubação e ao uso de antibiótico profilático relacionado à internação prévia no pré-transplante. Outros fatores de risco importantes que podem estar associados ao desenvolvimento de pneumonia precoce no pós-transplante de fígado incluem idade, MELD pré-, internamento prévio à cirurgia, tempo de isquemia fria e tempo de ventilação mecânica.

\section{ABSTRACT}

Purpose: To identify the main risk factors related to pneumonia in early pre-liver transplant surgery. Methods: A control case study performed from June to December of 2014 with patients submitted to liver transplant in a medical teaching hospital in Fortaleza, state of Ceará. It was included in the study patients of both genders submitted to liver transplant, and those presenting insufficient data in their medical records which would damage the analysis of data, those who died until the 5th post-surgery day, and those who were submitted to re-transplant due to primary dysfunction of the graft were excluded. The data collection was performed from June to December of 2014 by prospective analysis of the records from every patient submitted to liver transplant during that period. The records were analyzed in the pre, trans-, and post-surgery periods up to their hospital discharge Results: The sampling was composed by 52 patients being $69.2 \%$ male with average age of 55 years $( \pm 12.5)$. Different etiologies were found from which the most predominant were viral hepatitis $(51.9 \%)$ and alcoholic cirrhosis (21.1\%). Participants in the sampling presented an average MELD score of $22( \pm 4.8)$. The potential risk factors to develop early pneumonia in the liver post-transplantation by bivariate analysis include age $(p=0.09)$, pre-surgery MELD $(p=0.22)$, pre-hospitalization to the surgery $(p=0.09)$, time of cold ischemia $(p=0.22)$, and time of mechanical ventilation $(p=0.08)$. In the multivariate analysis, the independent risk factors were higher time in intensive care unit $(p=0.002)$, need for reintubation $(p=0.002)$, and use of a prophylactic antibiotic other than Ampicilin + Sulbactam (routine prophylactic antibiotic in the service) $(p=0.02)$. Conclusion: Pneumonia is a frequent pulmonary complication with a high mortality rate in the early liver post-transplant period. Such complication can be associated with several pre-, trans- and post-surgery factors.

Keywords: Risk Factors; Liver Transplantation; Pneumonia. 


\section{REFERÊNCIAS}

11. Galant LH, Forgiarini LAJr, Dias AS, Marroni CA. Condição funcional, força muscular respiratória e qualidade de vida em pacientes cirróticos. Rev. bras. fisioter. 2012;16(1):30-4.

2. Registro Brasileiro de Transplantes. Ano $X X, n^{\circ} 4$. Janeiro/ Dezembro, 2014, p. 3-10.

3. Baccaro LF, Boin Ide F, Costa-Paiva L, Pinto-Neto AM. Quality of life and menopausal symptoms in women with liver transplants. Rev. Bras. Ginecol. Obstet. 2013;35(3): 103-10.

4. Salvalaggio $\mathrm{P}$, Afonso RC, Ferraz BHN. Uma proposta para graduar a gravidade de disfunção precoce do enxerto após o transplante de fígado. Einstein (São Paulo). 2013;11(1):23-31.

5. Batista TP, Sabat BD, Melo PSV, et. al. Emprego do escore MELD para a predição da sobrevivência pós-transplante hepático. Rev. Col. Bras. Cir. 2012;39(2):105-11.

6. Mendes KDS, Rossin FM, Ziviani LC, et. al. Necessidades de informação de candidatos ao transplante de fígado: o primeiro passo do processo ensino-aprendizagem. Rev Gaúcha Enferm. 2012;33(4):94-102.

7. Coelho JCU, Trubian PS, Freitas ACT, et. al. Comparação entre o custo do transplante hepático cadavérico e o intervivos. Rev. Assoc. Med. Bras. 2005;51(3):158-63.

8. Feltracco P, Carollo C, Barbieri S, et. al. Early respiratory complications after liver transplantation. World J Gastroenterol. 2013;19(48):9271-81.

9. Conselho Nacional de Saúde (Brasil), Resolução n 466, de 12 de Dezembro de 2012. Aprova diretrizes e normas regulamentadoras de pesquisa envolvendo seres humanos. Diário Oficial da União 13 jun 2013; seção 1.

10. Ministério da Saúde (Brasil), Agência Nacional de Vigilância Sanitária (ANVISA). Série segurança do paciente e qualidade em serviços de saúde. Brasília: Ministério da Saúde; 2013. 79p.

11. Rothman K, Greenland S, Lash T. Modern Epidemiology. 3rd. Philadelphia: Lippincott Williamns \& Wilkins, 2008.

12. Glanemann M, Kaisers U, Langrehr JM, et. al. Incidence and indications for reintubation during postoperative care following orthotopic liver transplantation. J Clin Anesth. 2001;13(5):377-82.

13. Huang CT, Lin HC, Chang SC, Lee WC. Pre-operative risk factors predict post-operative respiratory failure after liver transplantation. PloS one. 2011;6(8):22689.

14. Freire MP. Infecções hospitalares em pacientes submetidos a transplante de fígado: fatores de risco relacionados ao doador. Dissertação [Mestrado em ciências médicas]. São Paulo: Universidade de São Paulo; 2006. Disponível em: http://www.teses.usp.br/teses/disponiveis/5/5134/tde03052007-110317/pt-br.php.
15. Qin J, Xu J, Dong Y, et. al. High-resolution CT findings of pulmonary infections after orthotopic liver transplantation in 453 patients. Br J Radiol. 2012;85(1019):959-65.

16. Concejero AM, Yong CC, Chen CL, et. al. Solitary pulmonary nodule in the liver transplant candidate: importance of diagnosis and treatment. Liver Transpl 2010;16(6):760-6.

17. Saner FH, Akkiz H, Canbay A. Infectious complications in the early postoperative period in liver transplant patients. Minerva Gastroenterol Dietol. 2010;56(3):355-66.

18. Golfieri R, Giampalma E, Labatti AM, et. al. Pulmonary complications of liver transplantation: radiological appearance and statistical evaluation of risk factors in 300 cases.Eur Radiol 2000;10(7):1169-83.

19. Benson AB, Burton JR, Austin GL, et. al. Differential effects of plasma and red blood cell transfusions on acute lung injury and infection risk following liver transplantation. Liver Transplantation. 2011;17(2):149-58.

20.Pirat A, Ozgur S, Torgay A, et. al. Risk factors for postoperative respiratory complications in adult liver transplant recipients. Transplant Proc. 2004;36(1):218-220.

21. Romero FA, Razonable RR. Infections in liver transplant recipients. World J Hepatol. 2011;3(4):83-92.

22.Zolfaghari PS, Wyncoll DL. The tracheal tube: gateway to ventilator-associated pneumonia. Crit Care. 2011;15(5):31017.

23.Lin YH, Cai ZS, Jiang Y, et al. Perioperative risk factors for pulmonary complications after liver transplantation. Journal of International Medical Research.2010;38(5):1845-55.

24. Vandecasteele E, De Waele J, Vandijck D, et. al. Antimicrobial prophylaxis in liver transplant patients-a multicenter survey endorsed by the European Liver and Intestine Transplant Association. Transplant International. 2010;23(2):182-90.

25. Classen DC, Evans RS, Pestotnik, SL, et. al. The timing of prophylactic administration of antibiotics and the risk of surgical-wound infection. New England Journal of Medicine. 1992;326(5):281-86.

26. Chihara Y, Egawa H, Oga T, et. al. Predictive Factors for Reintubation following Noninvasive Ventilation in Patients with Respiratory Complications after Living Donor Liver Transplantation. PLoS One. 2013;8(12):e81417.

27. Bozbas SS, Eyuboglu FO, Ozturk EF, et. al. Pulmonary complications and mortality after liver transplant. Experimental and clinical transplantation: official journal of the Middle East Society for Organ Transplantation. 2008;6(4):264-70. 